\title{
Nuclear effects in neutrino-nucleus DIS and a probe for short-range correlations
}

\author{
Fei Huang $\oplus^{2, *} \mathrm{Ji} \mathrm{Xu},{ }^{1, \dagger}$ and Xing-Hua Yang $\oplus^{3, \$}$ \\ ${ }^{1}$ School of Physics and Microelectronics, Zhengzhou University, Zhengzhou, Henan 450001, China \\ ${ }^{2}$ INPAC, Key Laboratory for Particle Astrophysics and Cosmology (MOE), \\ Shanghai Key Laboratory for Particle Physics and Cosmology, School of Physics and Astronomy, \\ Shanghai Jiao Tong University, Shanghai 200240, China \\ ${ }^{3}$ School of Physics and Optoelectronic Engineering, Shandong University of Technology, \\ Zibo, Shandong 255000, China
}

(Received 14 March 2021; accepted 29 June 2021; published 16 August 2021)

\begin{abstract}
We investigate charged-current neutrino-nucleus deep inelastic scattering with particular interest in the relationship of short-range correlation (SRC) and the EMC effect. The structure functions $F_{2}^{A}\left(x, Q^{2}\right)$, $x F_{3}^{A}\left(x, Q^{2}\right)$ and ratios of differential cross sections are presented where the nuclei $A$ are chosen to be carbon, iron, and lead. We propose a kind of universal modification function which would provide a nontrivial test of SRC universality on the platform of neutrino-nucleus scattering and improve our understanding of nucleon structure substantially.
\end{abstract}

DOI: $10.1103 /$ PhysRevD.104.033002

\section{INTRODUCTION}

As elementary particles in the standard model with no electric charge, neutrinos interact only via the weak interaction, which can be categorized by neutrino energies. Elastic scattering has been used to measure the nuclear charge distribution. Quasielastic scattering (QES) can serve as a normalization cross section when experimentalists are measuring the cross section ratios of neutrino scattering [1]. Resonance pion production (RES) is of critical importance to current and future neutrino oscillation experiments [2]. Neutrino-nucleus deep inelastic scattering (DIS) is sensitive to the nuclear quark distributions [3]. As the energy of neutrinos increases, the DIS process becomes dominant in neutrino-nucleus interactions.

One cannot determine the individual parton distribution functions (PDFs) through electron DIS experiments alone; information on the flavor decomposition of PDFs can be obtained by neutrino DIS experiments, which are crucial for global fits of PDFs, especially for the strange quark

\footnotetext{
* Corresponding author. fhuang@sjtu.edu.cn

Corresponding author. xuji_phy@zzu.edu.cn

Corresponding author. yangxinghua@sdut.edu.cn

Published by the American Physical Society under the terms of the Creative Commons Attribution 4.0 International license. Further distribution of this work must maintain attribution to the author(s) and the published article's title, journal citation, and DOI. Funded by SCOAP ${ }^{3}$.
}

distribution. A good knowledge of neutrino DIS is also a key factor for long-baseline experiments aiming at measuring the mixing angle and $\mathrm{CP}$-violating phase. In this process, a neutrino scatters with a quark inside a nucleon "deeply" (high-momentum transfer $Q^{2}$ ) at high energy, which destroys the nucleon. Because of the weak nature of neutrino interactions, the use of heavy nuclear targets is unavoidable; this brings complications, since correlations between nucleons, dubbed as nuclear effects, can affect observables in neutrino interaction. Nuclear effects are still not well understood in neutrino physics, and there has been renewed interest in investigating the nuclear effects in neutrino DIS processes for the following reasons: First, the data of current neutrino DIS measurements are valuable because the $F_{3}$ structure function can directly probe valence quark distributions. But these data contain non-negligible nuclear effects, since heavy nuclear targets such as iron and lead are used. Second, there is the issue of the NuTeV anomaly, which opens the question of factorization assumption in the nuclear environment - whether the neutrino DIS data could be combined with the charged-lepton DIS data to get better nuclear PDFs (NPDFs) [4-8]. This issue has attracted a lot of interest-Ref. [9] pointed out that this anomaly might be due to differences between the proton and the neutron caused by mean-field effects, and a nucleon-nucleon short-range correlation (SRC) explanation is also presented [10]. Third, nuclear effects are important in the analysis of high-energy neutrinos interacting with Earth matter-the total cross section is dominated by DIS off the partons from matter nucleons; therefore, the nuclear 
effects have significant impact on the predictions for the neutrino attenuation rates [11-16].

With such importance, neutrino DIS and nuclear effects have been explored worldwide—e.g., NuTeV [4], CHORUS [17], MINOS [18], and MINER $\nu \mathrm{A}$ [19] in recent years. They have analyzed neutrino DIS off different nuclear targets to measure structure functions, as well as differential and total cross sections. Reconciled with the charged-lepton DIS data and Drell-Yan (DY) dilepton production data, etc., the NPDFs can be extracted through global fitting [20,21]. One can resort to the global analysis of NPDFs with uncertainties in the CTEQ framework [22], the recent NPDF analyses [23], and EPPS16 [24] for more details.

The original idea of having nuclear effects in PDFs was driven by data in DIS measurements performed by the European Muon Collaboration (EMC) [25]. The initial expectation was that physics at the $\mathrm{GeV}$ scale would be insensitive to the nuclear binding effects, which are typically on the order of the several-MeV scale. However, the collaboration discovered that the per-nucleon deep inelastic structure function in iron is smaller than that of deuterium in the region $0.3<x<0.7$, where $x$ is the Bjorken variable. This phenomenon is known as the EMC effect and has been observed for a wide range of nuclei [26-30]. The EMC effect indicates that quark PDFs in nucleons are modified, breaking down the scale separation between nucleon structure and nuclear structure. Although the understanding of how the quark-gluon structure of a nucleon is modified by the surrounding nucleons has been brought to a whole new level, one should note that there is still no consensus as to the underlying dynamics that drives this effect even after more than three decades.

Currently, one of the leading approaches for describing the EMC effect is that nucleons bound in nuclei are unmodified, the same as "free" nucleons most of the time, but they are modified substantially when they fluctuate into SRC pairs. The SRC describes the probability that two nucleons are close in coordinate space, as a result of nontrivial nucleon-nucleon interactions in the nucleus. The connection between SRC and EMC effects has been extensively investigated in nuclear structure function measurements [31-34]. A linear relation between the magnitude of the EMC effect measured in electron DIS at $0.3<x<0.7$ and the SRC scale factor obtained from electron inelastic scattering at $x>1.5$ was proposed [35]; this striking linear relation suggests that the EMC effect and SRC correlation both stem from the same underlying physics, such as high local density and high-momentum nucleons in nuclei. This relation, if finally established, shall provide a unique method to study nuclear structure physics; see some of the recent developments in Refs. [36-39]. One of the key aspects of SRC is the universality, where the partonic structure from the correlated nucleon-nucleon pair is the same for all kinds of nuclei, and thus a universal modification function can be deduced. This function will be useful for testing QCD symmetry-breaking mechanisms and for distinguishing nuclear physics effects from beyond the standard-model effects in neutrino scattering experiments.

The robust linear correlation between the strength of the EMC and the SRC scale factor in nuclei indicates that possible modifications of the quark PDFs occur in nucleons which are inside SRC pairs. As illustrated above, the neutrino-nucleus DIS process is another ideal platform for testing nucleon structures and SRC interpretation of the EMC effect: it is sensitive to the quark distributions, especially for $u$ and $d$ quarks (antiquarks), and they contain crucial information on nuclear effects which should be distinguished from beyond-the-standard-model effects [40-46]. These can be studied by measuring three structure functions: $F_{1}\left(x, Q^{2}\right), F_{2}\left(x, Q^{2}\right)$, and $F_{3}\left(x, Q^{2}\right)[47,48]$. In this work, we will study charged-current neutrino-nucleus deep inelastic scattering where the nuclei $A$ are chosen to be ${ }^{12} \mathrm{C}$, ${ }^{56} \mathrm{Fe}$, and ${ }^{208} \mathrm{~Pb}$. The structure functions $F_{2}^{A}\left(x, Q^{2}\right)$ and $x F_{3}^{A}\left(x, Q^{2}\right)$ are calculated with the consideration of NPDFs by using EPPS16 parametrization [24], and our free nucleon baseline is CT14NLO [49]. The ratios of structure functions for nuclei and free nucleons as well as ratios of differential cross sections $d^{2} \sigma / d x d y$ are also presented for a better view of the EMC effect. A kind of universal modification functions were deduced in neutrino-nucleus DIS; these functions would provide a nontrivial test of SRC universality on the platform of neutrino-nucleus scattering.

The rest of this paper is organized as follows: The formalism and results of structure functions in neutrinonucleus DIS are presented in Sec. II. Differential cross sections $\left(d^{2} \sigma / d x d y\right)^{\nu A}$ and $\left(d^{2} \sigma / d x d y\right)^{\bar{\nu} A}$ are analyzed in Sec. III. Section IV is devoted to the discussion of universal modification functions. Finally, we summarize our paper and comment on future developments in Sec. V.

\section{FORMALISM FOR STRUCTURE FUNCTIONS IN NEUTRINO-NUCLEUS DIS}

Neutrino charged-current DIS depicts the process in which a neutrino scatters off a quark in the nucleon via the exchange of a $W^{ \pm}$boson whose momentum is $q$, producing a corresponding lepton and hadron in the final state, which can be written as

$$
\begin{aligned}
& \nu_{\ell}(k)+N(p) \rightarrow \ell\left(k^{\prime}\right)+X\left(p^{\prime}\right), \\
& \bar{\nu}_{\ell}(k)+N(p) \rightarrow \bar{\ell}\left(k^{\prime}\right)+X\left(p^{\prime}\right) .
\end{aligned}
$$

Here, $k$ is the momentum of the incoming neutrino (antineutrino), and $k^{\prime}$ indicates the momentum of the outgoing lepton (antilepton). The momenta of the initial nucleon and produced hadron are $p$ and $p^{\prime}$, respectively. These processes can be analyzed by the following Lorentz invariants: the Bjorken scaling variable $x \equiv Q^{2} /(2 p \cdot q)$, 


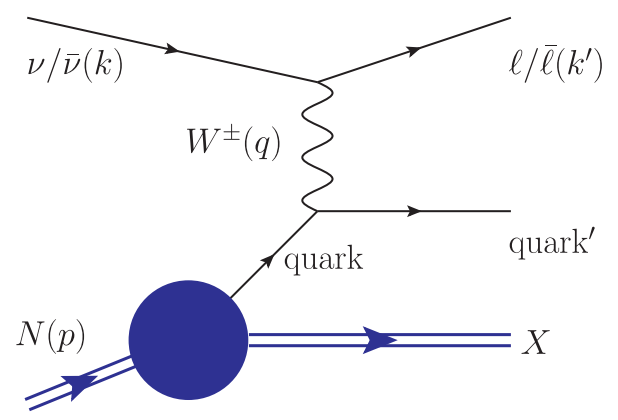

FIG. 1. Neutrino charged-current DIS process.

the inelasticity $y \equiv(2 p \cdot k) /(2 p \cdot q)$, and the negative squared four-momentum transfer $Q^{2} \equiv-q^{2}$. A schematic diagram of the neutrino charged-current DIS process is shown in Fig. 1.

The amplitude for neutrino charged-current interactions with the nucleon is described by the matrix element

$$
\mathcal{M}=\frac{G_{F} / \sqrt{2}}{1+Q^{2} / M_{W}^{2}} \bar{u}\left(k^{\prime}\right) \gamma^{\mu}\left(1-\gamma_{5}\right) u(k)\left\langle X\left|J_{\mu}^{C C}(0)\right| p\right\rangle,
$$

where $G_{F}$ is the Fermi coupling constant and $J_{\mu}^{C C}(0)$ is the weak charged current of the nucleon. The leptonic part can be calculated perturbatively, and the hadronic part becomes the hadron tensor $W_{\mu \nu}^{C C}$, which is then expressed by three structure functions in the expression of neutrino double differential cross sections [50]:

$$
\begin{aligned}
& \left(\frac{d \sigma}{d x d y}\right)^{\nu A}=\frac{G_{F}^{2} M_{N} E_{\nu}}{\pi\left(1+Q^{2} / M_{W}^{2}\right)^{2}}\left[F_{1}^{\nu A}\left(x, Q^{2}\right) x y^{2}+F_{2}^{\nu A}\left(x, Q^{2}\right)(1-y)+F_{3}^{\nu A}\left(x, Q^{2}\right) x y\left(1-\frac{y}{2}\right)\right], \\
& \left(\frac{d \sigma}{d x d y}\right)^{\bar{\nu} A}=\frac{G_{F}^{2} M_{N} E_{\bar{\nu}}}{\pi\left(1+Q^{2} / M_{W}^{2}\right)^{2}}\left[F_{1}^{\bar{\nu} A}\left(x, Q^{2}\right) x y^{2}+F_{2}^{\bar{\nu} A}\left(x, Q^{2}\right)(1-y)-F_{3}^{\bar{\nu} A}\left(x, Q^{2}\right) x y\left(1-\frac{y}{2}\right)\right] .
\end{aligned}
$$

Here $E_{\nu(\bar{\nu})}$ is the energy of the incoming neutrino (antineutrino), and $M_{N}$ is the nucleon mass. The expressions above can be further simplified by using the Callan-Gross relation $F_{2}^{\nu A}=2 x F_{1}^{\nu A}, F_{2}^{\bar{\nu} A}=2 x F_{1}^{\bar{\nu} A}$ [51]:

$$
\begin{aligned}
& \left(\frac{d \sigma}{d x d y}\right)^{\nu A}=\frac{G_{F}^{2} M_{N} E_{\nu}}{\pi\left(1+Q^{2} / M_{W}^{2}\right)^{2}}\left[\left(1-y+\frac{y^{2}}{2}\right) F_{2}^{\nu A}+x y\left(1-\frac{y}{2}\right) F_{3}^{\nu A}\right], \\
& \left(\frac{d \sigma}{d x d y}\right)^{\bar{\nu} A}=\frac{G_{F}^{2} M_{N} E_{\bar{\nu}}}{\pi\left(1+Q^{2} / M_{W}^{2}\right)^{2}}\left[\left(1-y+\frac{y^{2}}{2}\right) F_{2}^{\bar{\nu} A}-x y\left(1-\frac{y}{2}\right) F_{3}^{\bar{\nu} A}\right],
\end{aligned}
$$

with

$$
\left\{\begin{array}{l}
F_{2}^{\nu A}=\frac{Z}{A} F_{2}^{\nu p / A}+\frac{A-Z}{A} F_{2}^{\nu n / A} \\
F_{2}^{\bar{\nu} A}=\frac{Z}{A} F_{2}^{\bar{\nu} p / A}+\frac{A-Z}{A} F_{2}^{\bar{\nu} n / A} .
\end{array}\right.
$$

Here the explicit dependence on $x$ and $Q^{2}$ is omitted for brevity. We would like to discuss what kind of combinations of NPDFs can be constrained by the neutrino-nucleus DIS data; in the parton model, the structure functions for a nucleus with atomic mass $A$ and atomic number $Z$ can be expressed in terms of NPDFs (also suppressing the dependence on $x$ and $Q^{2}$ ):

$$
\begin{aligned}
F_{2}^{A} & =F_{2}^{\nu A}+F_{2}^{\bar{\nu} A}=\frac{Z}{A}\left(F_{2}^{\nu p / A}+F_{2}^{\bar{\nu} p / A}\right)+\frac{A-Z}{A}\left(F_{2}^{\nu n / A}+F_{2}^{\bar{\nu} n / A}\right) \\
& =2 x \frac{Z}{A}\left(f_{u}^{p / A}+f_{\bar{u}}^{p / A}+f_{d}^{p / A}+f_{\bar{d}}^{p / A}\right)+2 x \frac{A-Z}{A}\left(f_{u}^{n / A}+f_{\bar{u}}^{n / A}+f_{d}^{n / A}+f_{\bar{d}}^{n / A}\right),
\end{aligned}
$$

where $f_{i}^{p / A}$ represents the NPDFs of parton $i$ in a proton bound in nucleus $A$, and $f_{i}^{n / A}$ corresponds to a neutron. The NPDFs of strange and charm quarks are not considered here, since the contributions are very small in the EMC and kinematic regions where this work focuses.

Assuming isospin symmetry between bound neutrons and protons, one has

$f_{u, \bar{u}}^{n / A}=f_{d, \bar{d}}^{p / A}, \quad f_{d, \bar{d}}^{n / A}=f_{u, \bar{u}}^{p / A}, \quad f_{i^{\prime}}^{n / A}=f_{i^{\prime}}^{p / A}$ for other flavors.
Thus, the expression of the structure function in Eq. (5) can be further simplified as

$$
F_{2}^{A}=2 x\left(f_{u}^{p / A}+f_{d}^{p / A}+f_{\bar{u}}^{p / A}+f_{\bar{d}}^{p / A}\right)
$$

and similarly,

$$
F_{3}^{A}=2\left(f_{u}^{p / A}+f_{d}^{p / A}-f_{\bar{u}}^{p / A}-f_{\bar{d}}^{p / A}\right)
$$



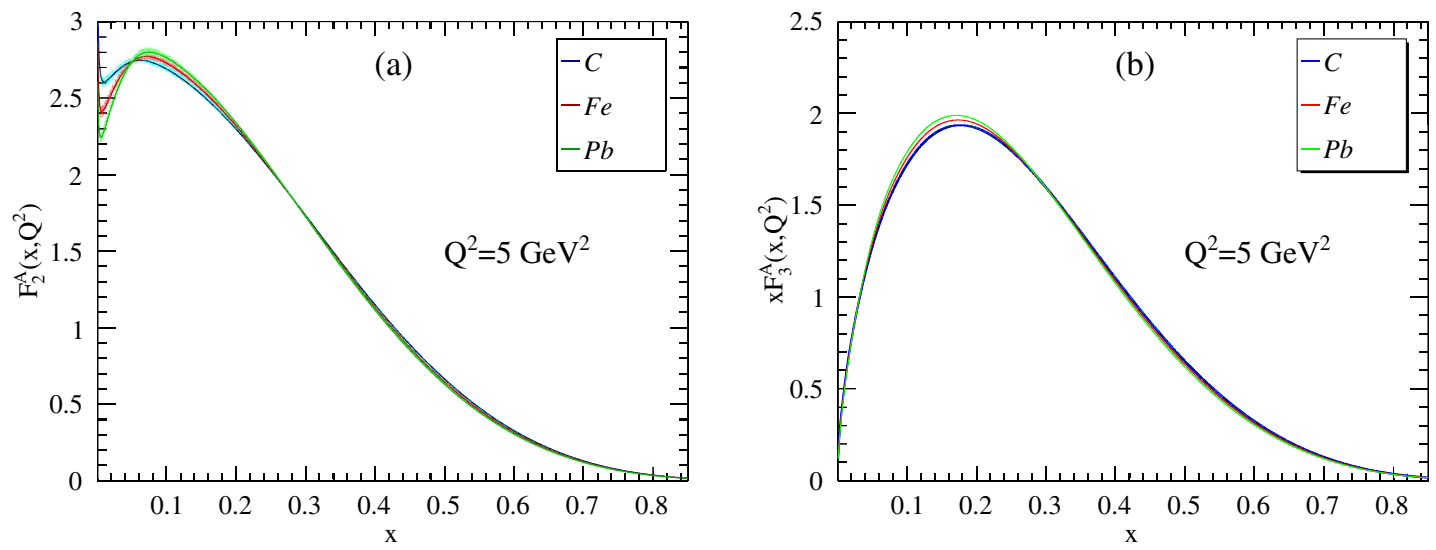

FIG. 2. Structure functions with EPPS16 parametrization of nuclear corrections for $\mathrm{C}, \mathrm{Fe}$, and $\mathrm{Pb}$ as functions of $x$ with $Q^{2}=5 \mathrm{GeV}^{2}$. $F_{2}\left(x, Q^{2}\right)$ and $x F_{3}\left(x, Q^{2}\right)$ are presented in plots (a) and (b), respectively. The shaded regions are uncertainties caused by NPDFs.

The NPDF $f_{i}^{p / A}\left(x, Q^{2}\right)$ can be defined relative to the free proton $\mathrm{PDF} f_{i}^{p}\left(x, Q^{2}\right)$ as [24]

$$
f_{i}^{p / A}\left(x, Q^{2}\right)=R_{i}^{A}\left(x, Q^{2}\right) f_{i}^{p}\left(x, Q^{2}\right),
$$

where $R_{i}^{A}\left(x, Q^{2}\right)$ is known as the nuclear modification factor. The free proton baseline is CT14NLO [49]. Figure 2 presents the structure functions $F_{2}^{A}\left(x, Q^{2}\right)$ and $x F_{3}^{A}\left(x, Q^{2}\right)$ with respect to $x$, with $Q^{2}$ fixed to $5 \mathrm{GeV}^{2}$, which is reachable in many neutrino-nucleus scattering experiments, and the uncertainties in this figure are caused by NPDFs. One can see that the lines in Fig. 2 are very close to each other; for a better illustration of the EMC effect, the ratios

$$
\begin{aligned}
& R\left(F_{2}^{A} ; x, Q^{2}\right)=\frac{f_{u}^{p / A}\left(x, Q^{2}\right)+f_{d}^{p / A}\left(x, Q^{2}\right)+f_{\bar{u}}^{p / A}\left(x, Q^{2}\right)+f_{\bar{d}}^{p / A}\left(x, Q^{2}\right)}{f_{u}^{p}\left(x, Q^{2}\right)+f_{d}^{p}\left(x, Q^{2}\right)+f_{\bar{u}}^{p}\left(x, Q^{2}\right)+f_{\bar{d}}^{p}\left(x, Q^{2}\right)}, \\
& R\left(F_{3}^{A} ; x, Q^{2}\right)=\frac{f_{u}^{p / A}\left(x, Q^{2}\right)+f_{d}^{p / A}\left(x, Q^{2}\right)-f_{\bar{u}}^{p / A}\left(x, Q^{2}\right)-f_{\bar{d}}^{p / A}\left(x, Q^{2}\right)}{f_{u}^{p}\left(x, Q^{2}\right)+f_{d}^{p}\left(x, Q^{2}\right)-f_{\bar{u}}^{p}\left(x, Q^{2}\right)-f_{\bar{d}}^{p}\left(x, Q^{2}\right)}
\end{aligned}
$$

are presented in Fig. 3.

\section{MODIFICATION FACTORS FOR $d^{2} \sigma / d x d y$}

Absolute neutrino flux is never known to be better than $20 \%-30 \%$, which makes total cross sections hard to

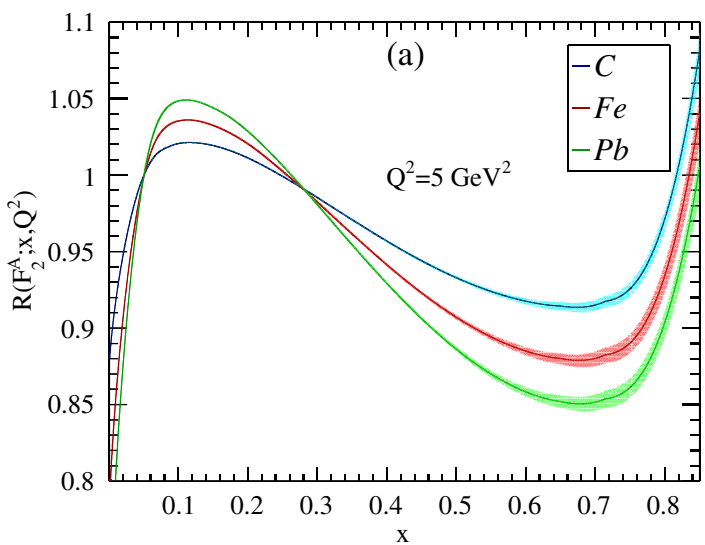

measure accurately, so experimentalists like to measure cross section ratios as well as the differential cross section, which is one of the least model-dependent measurements [52]. In the previous section, we have analyzed the structure functions of neutrino-nucleus DIS. In this section, we will

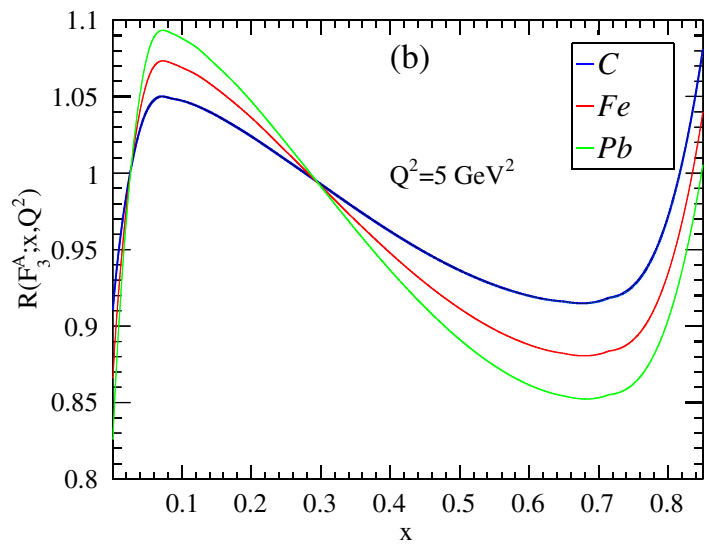

FIG. 3. The ratios (a) $R\left(F_{2}^{A} ; x, Q^{2}\right)$ and (b) $R\left(F_{3}^{A} ; x, Q^{2}\right)$ as functions of $x$ with $Q^{2}=5 \mathrm{GeV}^{2}$. The blue, red, and green lines correspond to $\mathrm{C}, \mathrm{Fe}$, and $\mathrm{Pb}$, respectively. As in Fig. 2, the shaded regions are uncertainties caused by NPDFs. 

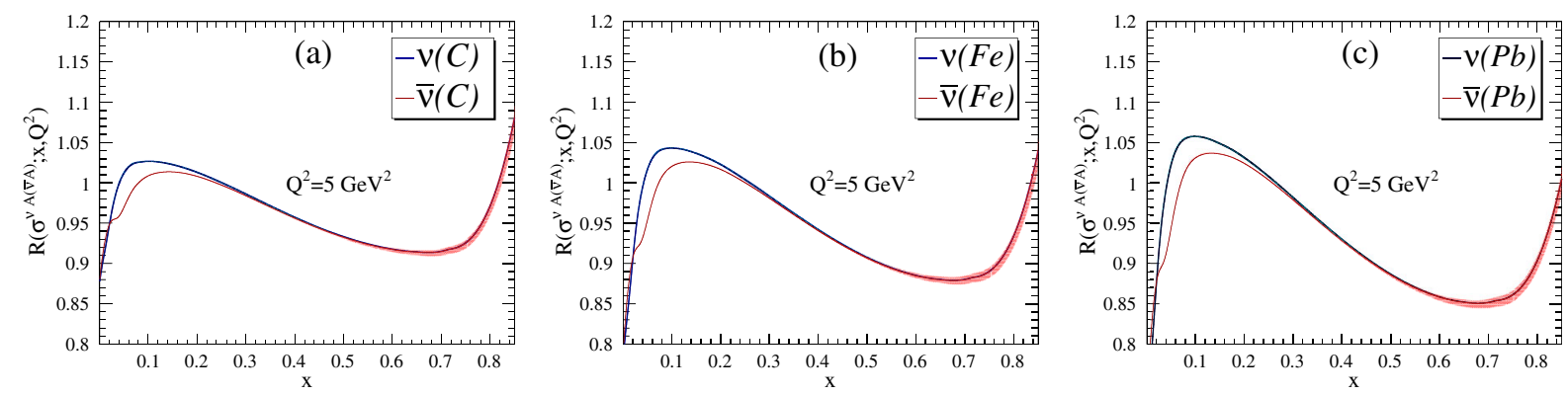

FIG. 4. Nuclear correction factor $R\left(\sigma^{\nu A(\bar{\nu} A)} ; x, Q^{2}\right)$ according to Eq. (11) in charged-current (a) $\nu(\bar{\nu}) \mathrm{C},(\mathrm{b}) \nu(\bar{\nu}) \mathrm{Fe}$, and (c) $\nu(\bar{\nu}) \mathrm{Pb}$ scattering. Here the shaded regions are presented as uncertainties.

compare differential cross sections of neutrino-nucleus DIS and neutrino-free nucleon DIS:

$R\left(\sigma^{\nu A} ; x, Q^{2}\right)=\left(\frac{d^{2} \sigma}{d x d y}\right)^{\nu A} /\left(\frac{d^{2} \sigma}{d x d y}\right)^{\nu \text { nucleon } / A}$,
$R\left(\sigma^{\bar{\nu} A} ; x, Q^{2}\right)=\left(\frac{d^{2} \sigma}{d x d y}\right)^{\bar{\nu} A} /\left(\frac{d^{2} \sigma}{d x d y}\right)^{\bar{\nu} \text { nucleon } / A}$,

where $R\left(\sigma^{\nu A(\bar{\nu} A)} ; x, Q^{2}\right)$ are called nuclear correction factors. The numerator in Eq. (11) can be derived from Eq. (4), and for the denominator we have used

$$
\begin{aligned}
& \left(\frac{d \sigma}{d x d y}\right)^{\nu \text { nucleon } / A} \\
& =\frac{G_{F}^{2} M_{N} E_{\nu}}{\pi\left(1+Q^{2} / M_{W}^{2}\right)^{2}}\left[\left(1-y+\frac{y^{2}}{2}\right) F_{2}^{\nu} \text { nucleon } / A\right. \\
& \left.\quad+x y\left(1-\frac{y}{2}\right) F_{3}^{\nu} \text { nucleon } / A\right] \\
& \left(\frac{d \sigma}{d x d y}\right)^{\bar{\nu} \text { nucleon } / A} \\
& =\frac{G_{F}^{2} M_{N} E_{\bar{\nu}}}{\pi\left(1+Q^{2} / M_{W}^{2}\right)^{2}}\left[\left(1-y+\frac{y^{2}}{2}\right) F_{2}^{\bar{\nu} \text { nucleon } / A}\right. \\
& \quad-x y\left(1-\frac{y}{2}\right) F_{3}^{\bar{\nu} \text { nucleon } / A]},
\end{aligned}
$$

with

$$
\left\{\begin{array}{l}
F_{2}^{\nu(\bar{\nu}) \text { nucleon } / A}=\frac{Z}{A} F_{2}^{\nu(\bar{\nu}) p}+\frac{A-Z}{A} F_{2}^{\nu(\bar{\nu}) n}, \\
F_{3}^{\nu(\bar{\nu}) \text { nucleon } / A}=\frac{Z}{A} F_{3}^{\nu(\bar{\nu}) p}+\frac{A-Z}{A} F_{3}^{\nu(\bar{\nu}) n} .
\end{array}\right.
$$

Our results are presented in Fig. 4 for $Q^{2}=5 \mathrm{GeV}^{2}$. The solid lines correspond to neutrinos, and the dashed lines to antineutrinos.

\section{UNIVERSALITY OF SRC}

The neutrino-nucleus DIS process is an ideal platform for testing the SRC interpretation of the EMC effect.
Motivated by the correlation between the EMC effect and the SRC scale factor and according to the universality of the SRC, we parametrize the $u$ and $d$ quark distributions in the EMC region as those for the structure function in Refs. [53,54] by assuming that all nuclear modifications originate from the nucleon-nucleon SRCs,

$f_{u_{V}\left(d_{V}\right)}^{p / A}\left(x, Q^{2}\right)=\frac{1}{Z}\left[Z f_{u_{V}\left(d_{V}\right)}^{p}\left(x, Q^{2}\right)+n_{s r c}^{A} \delta f_{u_{V}\left(d_{V}\right)}^{p}\left(x, Q^{2}\right)\right]$,

where $n_{s r c}^{A}$ represents the number of $n p$ pairs in nucleus $A$, notably the subscript $V$ in $f_{u_{V}}$, and $f_{d_{V}}$ means distributions of valence quarks, since experimental results pointed to the EMC effect being due to a change in the valence quark distributions. $\delta f_{u_{V}\left(d_{V}\right)}^{p}$ represents the difference between $u(d)$ valence quark distribution in the SRC pair and in the free proton. Substituting Eq. (12) into Eq. (9), we have

$$
\frac{\delta f_{u_{V}\left(d_{V}\right)}^{p}\left(x, Q^{2}\right)}{f_{u_{V}\left(d_{V}\right)}^{p}\left(x, Q^{2}\right)}=\frac{R_{u_{V}\left(d_{V}\right)}^{A}\left(x, Q^{2}\right)-1}{n_{s r c}^{A} / Z_{A}} .
$$

The left-hand side of the equation above is assumed to be nucleus-independent, meaning that the right-hand side should be a universal function which does not depend on the type of nucleus. Therefore, we can parametrize the quark distribution in $A$ in terms of that in $B$ :

$$
\begin{aligned}
R_{u_{V}\left(d_{V}\right)}^{A}\left(x, Q^{2}\right) & =\frac{n_{s r c}^{A} / Z_{A}}{n_{s r c}^{B} / Z_{B}}\left(R_{u_{V}\left(d_{V}\right)}^{B}\left(x, Q^{2}\right)-1\right)+1 \\
& =\frac{a_{2}^{A}}{a_{2}^{B}} \frac{Z_{B}}{Z_{A}} \frac{A_{A}}{A_{B}}\left(R_{u_{V}\left(d_{V}\right)}^{B}\left(x, Q^{2}\right)-1\right)+1 .
\end{aligned}
$$

Here, $a_{2}^{A}=\left(n_{s r c}^{A} / A\right) /\left(n_{s r c}^{d} / 2\right)$ is the SRC ratio of nucleus $A$ with respect to that of a deuteron. This ratio can be measured through the nuclear structure functions at the $x>$ 1.5 region $[35,55,56]$.

Based on the EPPS16 parametrization of quark NPDFs for carbon and Eq. (14), one can get quark NPDFs for iron and lead. With these distributions, the universality of SRC 

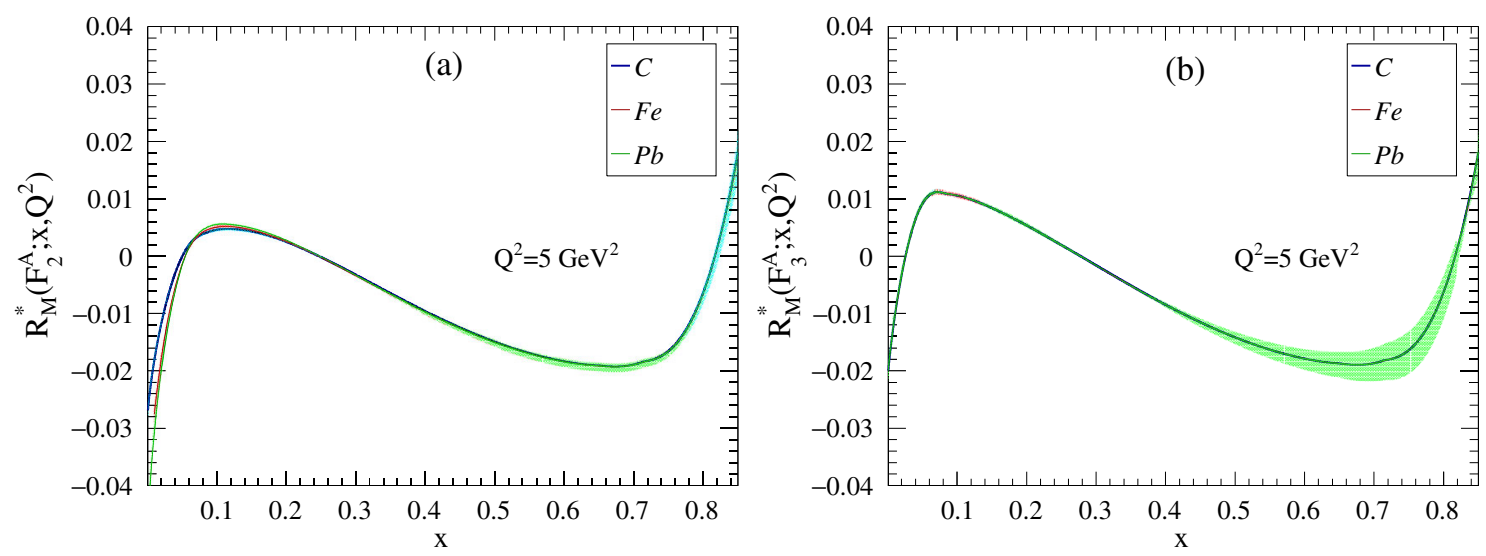

FIG. 5. Universality of EMC effects shown in the structure functions with the theoretical assumption in Eq. (14), here $Q^{2}=5 \mathrm{GeV}^{2}$. The nucleus-independent (universal) behavior of the SRC modification is clearly observed. The shaded regions are uncertainties caused by NPDFs and the scaling factor $a_{2}$.

can be illustrated more specifically by introducing one kind of universal modification functions whose functionality is normalizing the ratios defined in Eq. (10) by the respective SRC factors

$$
\begin{aligned}
& R_{M}^{*}\left(F_{2}^{A} ; x, Q^{2}\right)=\frac{2 Z_{A}}{A_{A}} \frac{R^{*}\left(F_{2}^{A} ; x, Q^{2}\right)-1}{a_{2}^{A}}, \\
& R_{M}^{*}\left(F_{3}^{A} ; x, Q^{2}\right)=\frac{2 Z_{A}}{A_{A}} \frac{R^{*}\left(F_{3}^{A} ; x, Q^{2}\right)-1}{a_{2}^{A}},
\end{aligned}
$$

where the $R^{*}$ marked with an asterisk is used to indicate that it is derived from Eq. (14)-i.e., from the assumption that all nuclear modifications originate from the SRCs in the EMC region. $R_{M}^{*}\left(F_{2}^{A} ; x, Q^{2}\right)$ and $R_{M}^{*}\left(F_{3}^{A} ; x, Q^{2}\right)$ are plotted in Fig. 5; the shaded regions are uncertainties which are mainly caused by the scaling factor $a_{2}$, whose contributions are introduced through Eq. (15). We can clearly see that the three curves of normalized ratios shrink almost into one single line at $0.3<x<0.7$; this is a remarkable prediction from the universality of the SRC contributions. Besides, Fig. 3 already shows the ratios $R\left(F_{2}^{A} ; x, Q^{2}\right)$ and $R\left(F_{3}^{A} ; x, Q^{2}\right)$, and they can also be normalized by Eq. (15), denoted as $R_{M}$. The results of $R_{M}\left(F_{2}^{A} ; x, Q^{2}\right)$ and $R_{M}\left(F_{3}^{A} ; x, Q^{2}\right)$ are shown in Fig. 6.

The predicted nucleus-independent universal behavior by the SRC-driven EMC model can be illustrated more clearly if we put these results together. The ratios $R$ defined in Eq. (10), the universal modification function $R_{M}$ with EPPS16 parametrization, and the universal modification function $R_{M}^{*}$ with the theoretical assumption have been presented in Fig. 7. One can clearly find that after they are normalized by the respective SRC factors, the ratios of different nuclei tend to shrink substantially; this observation is applicable to both $R_{M}$ and $R_{M}^{*}$. Besides this, the shapes of $R_{M}$ are consistent with $R_{M}^{*}$. This consistency supports the theoretical assumption in our paper and indicates that the EMC effect is mainly caused by SRC pairs in the EMC region. Figure 7 shows a nontrivial test of SRC universality.
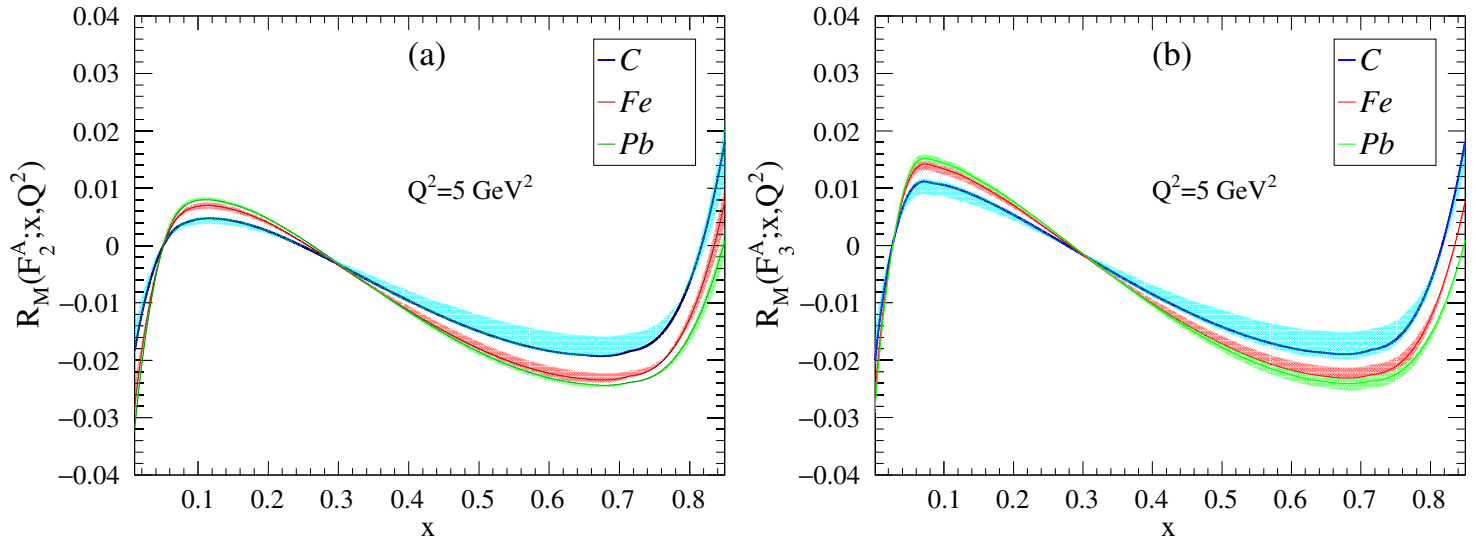

FIG. 6. Universality of EMC effects shown in the structure functions with EPPS16 parametrization, here $Q^{2}=5$ GeV ${ }^{2}$ As in Fig. 5 , the shaded regions are uncertainties caused by NPDFs and the scaling factor $a_{2}$. 

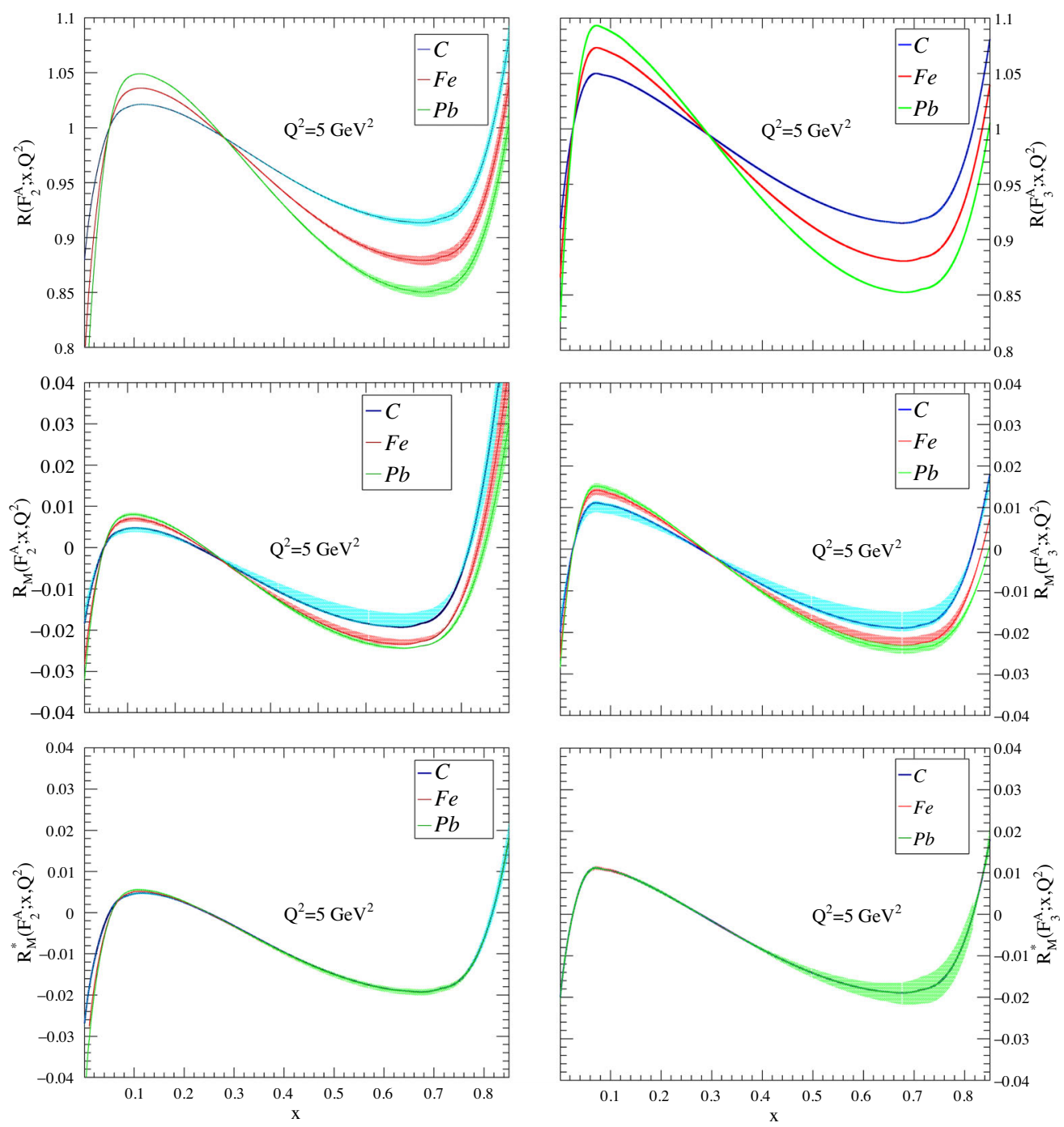

FIG. 7. Results of $R\left(F_{2}^{A}\left(F_{3}^{A}\right) ; x, Q^{2}\right)$ (the first row), $R_{M}\left(F_{2}^{A}\left(F_{3}^{A}\right) ; x, Q^{2}\right)$ (the second row), and $R_{M}^{*}\left(F_{2}^{A}\left(F_{3}^{A}\right) ; x, Q^{2}\right)$ (the third row) are presented together in this figure for convenience of comparison.

\section{CONCLUSION}

In summary, we have studied charged-current neutrinonucleus deep inelastic scattering with particular interests in the relationship of SRC and the EMC effect. The ratios of structure functions $F_{2}^{A}\left(x, Q^{2}\right), x F_{3}^{A}\left(x, Q^{2}\right)$ and differential cross sections are presented. We also show the consistency of EMC data and the SRC interpretation of the EMC effect based on the assumption of SRC universality. From these results, one can come to the conclusion that the EMC effect in different nuclei can be described by the abundance of SRC pairs and the proposed modification functions are in fact universal. These universal modification functions presented here provide a new clue to understand the fundamental aspects of QCD.

We would like to emphasize that the data used in EPPS16 include not only neutrino-nucleus DIS, but also charged-lepton-nucleus DIS, Drell-Yan dilepton production, and inclusive pion production, etc. To purely test the universality of SRC through neutrino-nucleus DIS, more data and new observables were called for. These new data will improve our understanding of nucleon structure substantially.

\section{ACKNOWLEDGMENTS}

The authors are grateful to Professor Wei Wang for inspiring discussions and valuable comments. F. H. is supported in part by the Natural Science Foundation of China under Grants No. 11735010, No. U2032102, No. 11653003, and by the Natural Science Foundation of Shanghai under Grant No. 15DZ2272100. J.X. is supported in part by the National Natural Science Foundation of China under Grant No. 12047545. 
[1] P. A. Zyla et al. (Particle Data Group), Prog. Theor. Exp. Phys. (2020), 083C01.

[2] C. Giganti, S. Lavignac, and M. Zito, Prog. Part. Nucl. Phys. 98, 1 (2018).

[3] O. Hen, G. A. Miller, E. Piasetzky, and L. B. Weinstein, Rev. Mod. Phys. 89, 045002 (2017).

[4] M. Tzanov et al. (NuTeV Collaboration), Phys. Rev. D 74, 012008 (2006).

[5] I. Schienbein, J. Y. Yu, C. Keppel, J. G. Morfin, F. Olness, and J. F. Owens, Phys. Rev. D 77, 054013 (2008).

[6] I. Schienbein, J. Y. Yu, K. Kovarik, C. Keppel, J. G. Morfin, F. Olness, and J. F. Owens, Phys. Rev. D 80, 094004 (2009).

[7] K. Kovarik, I. Schienbein, F. I. Olness, J. Y. Yu, C. Keppel, J. G. Morfin, J. F. Owens, and T. Stavreva, Phys. Rev. Lett. 106, 122301 (2011).

[8] H. Paukkunen and C. A. Salgado, J. High Energy Phys. 07 (2010) 032.

[9] I. C. Cloet, W. Bentz, and A. W. Thomas, Phys. Rev. Lett. 102, 252301 (2009).

[10] B. Schmookler et al. (CLAS Collaboration), Nature (London) 566, 354 (2019).

[11] A. Garcia, R. Gauld, A. Heijboer, and J. Rojo, J. Cosmol. Astropart. Phys. 09 (2020) 025.

[12] V. Bertone, R. Gauld, and J. Rojo, J. High Energy Phys. 01 (2019) 217.

[13] X. Wang and S. Zhou, Nucl. Phys. B950, 114867 (2020).

[14] Z. Z. Xing and J. Y. Zhu, Nucl. Phys. B949, 114803 (2019).

[15] Y. F. Li, Y. Wang, and Z. Z. Xing, Chin. Phys. C 40, 091001 (2016).

[16] Z. Z. Xing, Phys. Rev. D 63, 073012 (2001).

[17] G. Onengut et al. (CHORUS Collaboration), Phys. Lett. B 632, 65 (2006).

[18] P. Adamson et al. (MINOS Collaboration), Phys. Rev. D 81, 072002 (2010).

[19] J. Mousseau et al. (MINER $\nu$ A Collaboration), Phys. Rev. D 93, 071101 (2016).

[20] K. J. Eskola, Nucl. Phys. A910-911, 163 (2013).

[21] H. Paukkunen, Nucl. Phys. A926, 24 (2014).

[22] K. Kovarik, A. Kusina, T. Jezo, D. B. Clark, C. Keppel, F. Lyonnet, J. G. Morfin, F. I. Olness, J. F. Owens, I. Schienbein et al., Phys. Rev. D 93, 085037 (2016).

[23] E. P. Segarra, T. Ježo, A. Accardi, P. Duwentäster, O. Hen, T. J. Hobbs, C. Keppel, M. Klasen, K. Kovařík, and A. Kusina et al., Phys. Rev. D 103, 114015 (2021).

[24] K. J. Eskola, P. Paakkinen, H. Paukkunen, and C. A. Salgado, Eur. Phys. J. C 77, 163 (2017).

[25] J. J. Aubert et al. (European Muon Collaboration), Phys. Lett. B 123, 275 (1983).

[26] M. Arneodo et al. (European Muon Collaboration), Phys. Lett. B 211, 493 (1988).

[27] M. Arneodo et al. (European Muon Collaboration), Nucl. Phys. B333, 1 (1990).

[28] D. Allasia et al. (New Muon (NMC) Collaboration), Phys. Lett. B 249, 366 (1990).

[29] J. Gomez, R. G. Arnold, P. E. Bosted, C. C. Chang, A. T. Katramatou, G. G. Petratos, A. A. Rahbar, S. E. Rock, A. F. Sill, Z. M. Szalata et al., Phys. Rev. D 49, 4348 (1994).
[30] J. Seely, A. Daniel, D. Gaskell, J. Arrington, N. Fomin, P. Solvignon, R. Asaturyan, F. Benmokhtar, W. Boeglin, B. Boillat et al., Phys. Rev. Lett. 103, 202301 (2009).

[31] K. S. Egiyan et al. (CLAS Collaboration), Phys. Rev. Lett. 96, 082501 (2006).

[32] O. Hen, E. Piasetzky, and L. B. Weinstein, Phys. Rev. C 85, 047301 (2012).

[33] O. Hen, M. Sargsian, L. B. Weinstein, E. Piasetzky, H. Hakobyan, D. W. Higinbotham, M. Braverman, W. K. Brooks, S. Gilad, K. P. Adhikari et al., Science 346, 614 (2014).

[34] M. Duer et al. (CLAS Collaboration), Nature (London) 560, 617 (2018).

[35] L. B. Weinstein, E. Piasetzky, D. W. Higinbotham, J. Gomez, O. Hen, and R. Shneor, Phys. Rev. Lett. 106, 052301 (2011).

[36] J. W. Chen, W. Detmold, J. E. Lynn, and A. Schwenk, Phys. Rev. Lett. 119, 262502 (2017).

[37] J. E. Lynn, D. Lonardoni, J. Carlson, J. W. Chen, W. Detmold, S. Gandolfi, and A. Schwenk, J. Phys. G 47, 045109 (2020).

[38] J. Xu and F. Yuan, Phys. Lett. B 801, 135187 (2020).

[39] Y. Hatta, M. Strikman, J. Xu, and F. Yuan, Phys. Lett. B 803, 135321 (2020).

[40] M. E. Peskin and D. V. Schroeder, An Introduction to quantum field theory.

[41] D. Grover, K. Saraswat, P. Shukla, and V. Singh, Phys. Rev. C 98, 065503 (2018).

[42] O. Hen, D. W. Higinbotham, G. A. Miller, E. Piasetzky, and L. B. Weinstein, Int. J. Mod. Phys. E 22, 1330017 (2013).

[43] S. Y. Li, Z. G. Si, and X. H. Yang, Phys. Lett. B 795, 49 (2019).

[44] Z. G. Si, X. H. Yang, and S. Zhou, Chin. Phys. C 41, 113105 (2017).

[45] F. Huang, H. L. Li, S. Y. Li, Z. G. Si, W. Su, and Z. J. Yang, Chin. Phys. C 42, 033103 (2018).

[46] W. Yang and F. Huang, Int. J. Mod. Phys. A 35, 2050212 (2020).

[47] S. A. Kulagin and A. V. Sidorov, Eur. Phys. J. A 9, 261 (2000).

[48] D. J. Broadhurst, A. L. Kataev, and C. J. Maxwell, Phys. Lett. B 590, 76 (2004).

[49] S. Dulat, T. J. Hou, J. Gao, M. Guzzi, J. Huston, P. Nadolsky, J. Pumplin, C. Schmidt, D. Stump, and C. P. Yuan, Phys. Rev. D 93, 033006 (2016).

[50] M. Hirai, S. Kumano, and K. Saito, AIP Conf. Proc. 1189, 269 (2009).

[51] C. G. Callan, Jr. and D. J. Gross, Phys. Rev. Lett. 22, 156 (1969).

[52] M. Tzanov, AIP Conf. Proc. 1222, 243 (2010).

[53] L. L. Frankfurt, M. I. Strikman, D. B. Day, and M. Sargsian, Phys. Rev. C 48, 2451 (1993).

[54] E. P. Segarra, A. Schmidt, T. Kutz, D. W. Higinbotham, E. Piasetzky, M. Strikman, L. B. Weinstein, and O. Hen, Phys. Rev. Lett. 124, 092002 (2020).

[55] N. Fomin, J. Arrington, R. Asaturyan, F. Benmokhtar, W. Boeglin, P. Bosted, A. Bruell, M. H. S. Bukhari, M. E. Christy, E. Chudakov et al., Phys. Rev. Lett. 108, 092502 (2012).

[56] J. Arrington, A. Daniel, D. Day, N. Fomin, D. Gaskell, and P. Solvignon, Phys. Rev. C 86, 065204 (2012). 